\section{Ukrainian Journal \\ of Educational Studies and Information Technology}

ISSN: 2521-1234 online

Ukr. J. of Educ. Stud. and Inf. Technol, 7(1), 1-18

doi: $\underline{10.32919 / \text { uesit.2019.01.01 }}$

Vol. 7, Issue 1, 2019

UDC 378.147.811

\title{
To the question of foreign-language training of undergraduates of power specialities in technical establishments of higher education of Ukraine
}

\author{
Nataliia Bilan \\ Separated Subdivision of National University of Live and Environmental Sciences of Ukraine \\ "Berezhany Agrotechnical Institute", \\ Berezhany, Ukraine
}

\begin{tabular}{l} 
ARTICLE INFO \\
\hline History: \\
Received: 29.01 .2019 \\
Accepted: 05.03 .2019 \\
Published online: 20.03 .2019 \\
\hline Keywords: \\
foreign language training \\
foreign language \\
foreign competence \\
undergraduate \\
power-engineering specialist \\
technical establishments of higher \\
education \\
types of speech activity
\end{tabular}

(c) N. Bilan

This work is licensed under a "CC BY 4.0" license.

\section{ABSTRACT}

In the article the current state of foreign linguistic preparation of future power-engineering specialists in technical institutions of higher education is investigated and analysed. On the basis of the theoretical review of scientific and pedagogical literature it is presented the positions of researchers and the personal treatment of the designated process are offered. Foreign linguistic preparation is an inseparable component of purposeful process of vocational training of undergraduates of power specialties which is directed to the achievement of the educational, developing, educator, world outlook, professional purpose, and is characterized by acquisition of foreign-language and background knowledge, accompanies formation of practical abilities and skills, foreign-language competence and professional and personal qualities of future power engineering specialists. On the basis of experimental work results, it is defined and proved forms of the organization and implementation of foreign-language training of undergraduates in technical institutions of higher education which provide attending of the combined classes in the scientific discipline «Business English», language courses, membership in scientific circles, participation in student's conferences, creative competitions, passing of educational practices and internship abroad. Taking into account such criteria as the organization of educational process, material and technical resources, educational and methodical providing, the contingent of students, the international activity, - the condition of foreignlanguage training of undergraduates of power specialties in technical institutions of the higher education is estimated by a fivepoint system.

It is determined that foreign-language training of future power engineers is based on competent, personally focused, active, problematical and differentiated approaches realization of which is provided by the harmonious use of communicative, situational and 
group methods of training with innovative pedagogical technologies. On the basis of observation and poll the positive attitude and the considerable interest of undergraduates of power specialties in a learning of foreign languages and also difficulty which arise in the course of foreign-language preparation is revealed and proved ways of their overcoming. Behind the results of an empirical research, levels of formation of foreign-language competence of future power engineers are determined and their ranging behind receptive and productive types of colloquial activity is carried out. On the example of the theme «Official Journey to Germany», it is proved possibilities of complex development and improvement of skills of audition, reading, oral and written language of student's power engineering specialists. The reasons of mediocre level of competence in foreign languages by future power engineers are defined and the elimination of which will allow to avoid shortcomings of the organization and fulfilment of foreign language training of future power-engineers in the higher education establishments.

\title{
Zur Frage der fremdsprachlichen Ausbildung der Magister der energetischen Fachrichtungen an den technischen Hochschulen der Ukraine
}

\author{
Natalia Bilan \\ Getrennte Abteilung der Nationalen Universität für Lebens- und Umweltwissenschaften der Ukraine \\ "Das Bereshaner agrotechnische Institut", \\ Bereshany, Ukraine
}

\begin{abstract}
Abstrakt. Im vorliegenden Artikel ist der gegenwärtige Zustand der fremdsprachlichen Ausbildung der künftigen Ingenieur-Energetiker an den technischen Hochschulen erforscht und analysiert. Auf der Grundlage der theoretischen Übersicht wissenschaftlich-pädagogischer Literatur sind Stellungen zeitgenössischer Forscher dargelegt und eigene Deutung zum Prozess dargeboten. Es wurden die Besonderheiten der Organisation und Verwirklichung der fremdsprachlichen Ausbildung von Erwerbern der Bildungsstufe "Magister" unter Berücksichtigung der Spezifik der Berufstätigkeit von Fachkräften des energetischen Bereiches erörtert. Dargelegt werden die Ergebnisse der empirischen Forschung zum aufgezeichneten Problem, auf deren Grundlage die Niveaus der angeeigneten fremdsprachlichen Kompetenz der Magisterstudenten-Energetiker nach rezeptiven und produktiven Arten der sprachlichen Aktivität ermittelt wurden: Hörverstehen, Lesen, Sprechen und Schreiben.
\end{abstract}

Schlüsselwörter: fremdsprachliche Ausbildung; Fremdsprache; fremdsprachliche Kompetenz; Magisterstudenten; zukünftige Ingenieur-Energetiker; technische Hochschulen; Arten der sprachlichen Aktivität.

\section{EINLEITUNG}

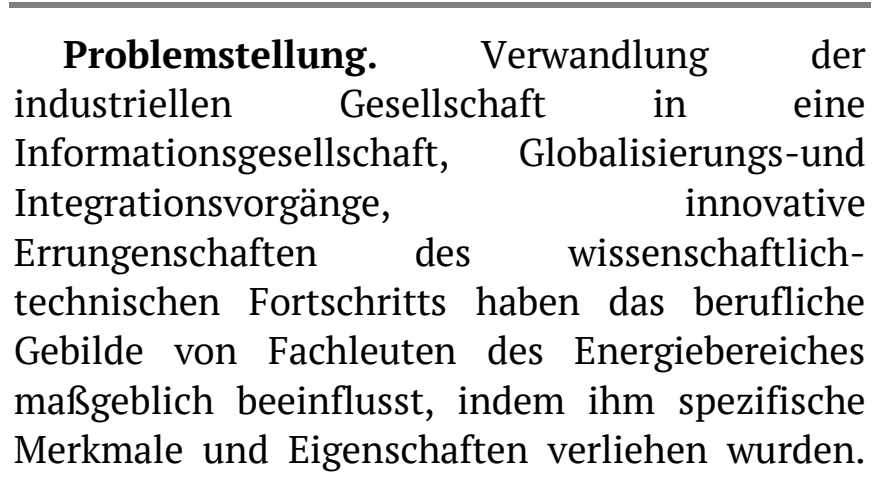

Heutzutage wird ein solcher IngenieurEnergetiker angefordert, der eine organisatorisch-verwaltungsmäßige, produktions-technologische, entwurfs- und konstruktionsmaßige, wissenschaftliche Forschungstätigkeit sachkundig ausübt und praktische Sprachfähigkeiten bzw. Sprachfertigkeiten in der Anwendung der fremdsprachlichen Kenntnisse im beruflichen Milieu besitzt. Eine Fremdsprache wird zu einem eigenartigen Mittel der Steigerung von 
Konkurrenzfähigkeit und Arbeitsbeschaffung von Absolventen der energetischen Fachrichtungen auf dem inländischen und ausländischen Arbeitsmarkt, zum Mittel der Verständigung und der erfolgreichen Zusammenarbeit mit Investoren und Kollegen aus Europa. An besonderer Aktualität gewinnt im Zusammenhang damit das Problem der Bildung der fremdsprachlichen Kompetenz der Magisterstudenten an den technischen Hochschulen, deren Lösung dank qualitativer Berufsausbildung zukünftiger Ingenieur-Energetiker unter Berücksichtigung der sozialen Anforderungen der Gesellschaft und des Bedarfs im energetischen Bereich möglich ist.

$\begin{array}{cccr}\text { Analyse der } & \text { letzten } & \text { Forschungen } & \text { und } \\ \text { Publikationen. } & \text { Das } & \text { Problem } & \text { der }\end{array}$
fremdsprachlichen Ausbildung der Studierenden an den technischen Hochschulen der Ukraine war Gegenstand vieler wissenschaftlicher Forschungen. Die Besonderheiten ihrer Organisation sind in den wissenschaftlichen Arbeiten von Z. Kornjewa, S. Kostryzjka, N. Schewerun, W. Trundjuk, O. Zabolotsjka, I. Zuenok dargelegt. Die Forscher vertreten verschiedene Gesichtspunkte und verstehen den Grundbegriff unserer Forschung unterschiedlich. Der Ansicht von Schumsjka (2015) nach ist die fremdsprachliche Ausbildung ein Mittel der Bildung des soziokulturellen Potenzials der Gesellschaft, welches unter Bedingungen aktiver Entwicklung der internationalen Beziehungen zum Hauptglied in der Entwicklung des Staates wird, denn eine der dringlichsten Aufgaben der europäischen Hochschulbildung ist Schaffung eines gemeinsamen Sprachraums und Stärkung der Aufmerksamkeit gegenüber dem fremdsprachlichen Bestandteil des Inhalts der Hochschulbildung (․ 205). Der Wissenschaftler Chomenko (2012) erachtet die fremdsprachliche Ausbildung als Bildungssegment, das eine führende Rolle im Werden eines innovativen Bildungssystems spielt, das auf den Beitritt zum Weltbildungsraum gerichtet ist, es wird durch die Suche solcher innovativen Wege des Herangehens und der Lernmethoden im Fremdsprachenunterricht unter Bedingungen der Humanisierung von Bildung gekennzeichnet, deren Umsetzung den zukünftigen Fachmann auf interkulturelles Zusammenwirken bringt ( $\underline{\text { S. 58) }}$ ). In ihrer Dissertation schlägt Schumejko (2017) folgende Definition der fremdsprachlichen Ausbildung vor - dies sei ein zielstrebiger dynamischer Vorgang, der durch Einheitlichkeit von Ziel, Struktur und Inhalt der Bildung gekennzeichnet ist; sie sieht das Erlernen einer Fremdsprache und Kultur unter Berücksichtigung theoretischer Grundlagen von «Gemeinsamer europäischer Referenzrahmen für Sprachen: lernen, lehren, beurteilen» (․ 17). Die Forscherin Nitenko (2015) betrachtet die fremdsprachliche Ausbildung als ein systematisches Beherrschen der Fremdsprachen auf Grund fachübergreifender Integration mit Berücksichtigung der individuellen Eigenschaften der Persönlichkeit. Dieses Beherrschen soll auf Erreichen eines solchen Niveaus der beruflichen fremdsprachlichen kommunikativen Kompetenz gerichtet werden, was interkulturelle und zwischenmenschliche Kommunikation zur Lösung berufsmäßiger Aufgaben in der multikulturellen, multilinqualen globalisierten Gesellschaft sicherstellt (․ 58).

Fremdsprachliche Ausbildung bedeutet einen vielseitigen Begriff, der alle Bestandteile der Berufsbildung umfasst, deren qualitativer Indikator maßgeblich von der Anwendung der innovativen pädagogischen Technologien im Erlernen der Fremdsprachen abhängt, in denen wissenschaftliche

Untersuchungen
T. Herasymtschuk,
M. Kuz,
O. Mazjuk,

M. Pradivljannyj, O. Schumskjyj, I. Stavytzjka, O. Zahorodna unternommen haben. Die Entwicklung der Sprachfertigkeiten und Bereitschaft zukünftiger Fachkräfte in ihrer Berufstätigkeit fremdsprachlich kommunizieren zu können haben D. Bubnowa, O. Kawerina, H. Krawtschuk, H. Kyslynsjka, O. Mosharowsjka, J. Nikolajenko, N. Rubel, L. Rusalkina erforscht. Die fremdsprachliche Kompetenz als Ergebnis der fremdsprachlichen Ausbildung der Erwerber von verschiedenen Fachrichtungen findet ihre Begründung in den wissenschaftlichen Arbeiten von D. Demtschenko, T. Herasymtschuk,
N. Mukutenko,
L. Nahornjuk,
A. Petrowa,
O. Tynkaljuk.

Jedoch ungeachtet einer Reihe von wissenschaftlichen Publikationen offen bleibt das Problem der fremdsprachlichen Ausbildung von Magisterstudenten der energetischen Fachrichtungen, mit deren Organisation und 
Verwirklichung das Endergebnis zusammenhängt - das auf Fähigkeit zukünftiger Ingenieure zurückgeführt wird, mit Kenntnissen einer Fremdsprache im Berufsleben praktisch umzugehen.

Das Ziel des Artikels besteht in der Erforschung und Analyse des Zustandes der fremdsprachlichen Ausbildung zukünftiger Ingenieur-Energetiker an den technischen Hochschulen, in der Begründung der Ergebnisse des bestimmten Vorgangs mit Feststellung der Stufen der Bildung fremdsprachlicher Kenntnisse, praktischer Fähigkeiten und Fertigkeiten der Magisterstudenten in rezeptiven und produktiven Arten der sprachlichen Aktivität.

\section{FORSCHUNGSMETHODIK}

Zur Feststellung des gegenwärtigen Zustandes der fremdsprachlichen Ausbildung zukünftiger Ingenieur-Energetiker an den technischen Hochschulen haben Methoden der empirischen Forschung Anwendung gefunden, insbesondere Befragen, Beobachten. Als Forschungsstationen dienten auf der feststellenden Etappe die Nationale Universität «Lvivsjka Politechnika», die Ternopiler Nationale technische Universität von Ivan Puluj, die getrennte Abteilung der Nationalen Universität für Lebens- und Umweltwissenschaften der Ukraine «Das Bereshaner agrotechnische Institut».

Um die Einstellung von Studierenden zum Fremdsprachenerwerb festzustellen, darunter auch die Bedingungen, die durch technische Hochschulen für die erfolgreiche Bildung der fremdsprachlichen Kompetenz geschaffen worden sind, wurde unter 266 Magisterstudenten der Studiengänge 141 «Elektroenergetik, Elektrotechnik und Elektromechanik», 143 «Atomenergetik», 144 «Wärmeenergetik» eine anonyme Umfrage durchgeführt. Zur Umfrage wurden auch 42 Hochschullehrer herangezogen, die das Unterrichten der Lernfächer «Berufsbezogene Fremdsprache», «Geschäftsfremdsprache», «Fachbezogene Fremdsprache», «Fachorientierte Fremdsprache» an den Fakultäten sicherstellen und die Berufsausbildung zukünftiger Fachleute im Energiezweig ausüben. Die Forschung wurde im zweiten Semester 2017-2018 und im ersten Semester 2018-2019 durchgeführt.

\section{FORSCHUNGSERGEBNISSE}

Auf Grund der Angaben, die man im Ergebnisse empirischer Forschung und eigener Erfahrung erhalten hat, wird die Analyse des gegenwärtigen Zustands der fremdsprachlichen Ausbildung von Magisterstudenten der energetischen Fachrichtungen an den technischen Hochschulen vorgelegt.

Die fremdsprachliche Ausbildung der Erwerber der zweiten Magisterstufe der Hochschulbildung beruht auf normativen Bildungsmaterialien und wird entsprechend den Herausforderungen der Deklaration von Bologna verwirklicht. Dazu gehören Gemeinsamer europäischer Referenzrahmen für Sprachen: lernen, lehren, beurteilen (Nikolajewa, 2003), Curriculum für kommunikatives Berufsenglisch (Bakajewa, Borysenko, Sujenok, Iwanischtschewa, Klymenko, Kozymyrska, Kostrytzjka, Skrypnyk, Todorowa, \& Chodzewa, 2005), Rahmencurriculum für den Studienbegleitenden Deutschunterricht an den ukrainischen Hochschulen (Azzolini, Amelina, Hamanjuk, \& Shdanowa, 2014). Sie sind die Hauptrichtlinien für technische Hochschulen, die Bezug auf Organisation und Verwirklichung der sprachlichen Ausbildung von Studierenden nehmen, und für Fremdsprachenlehrer sind sie eigenartige Wegweiser bei der Ausarbeitung von Arbeitsprogrammen, methodischen Hinweisen bezüglich der Aneignung des Fachwortschatzes und der Fachausdrücke, der Annotation und des Referierens der wissenschaftlich-technischen Quellen eines ausländischen Verlags, etc., der Lehrbücher, der Kriterien zur Bewertung fremdsprachlicher Kenntnisse für laufende und abschließende Kontrolle.

An den technischen Hochschulen wird die Ausbildung der Magisterstudenten des Wissenszweigs 14 «Elektrisches Engineering» der Studiengänge Elektrotechnik und Elektromechanik», 143 «Atomenergetik», 144 «Wärmeenergetik» durch das Erlernen des Lehrfachs «Geschäftsfremdsprache» / «Fachbezogene Fremdsprache» / «Fachorientierte Fremdsprache» sichergestellt, das zum Verzeichnis der Wahlfächer des allgemeinbildenden Zyklus gehört. Die kognitive Bildungsaktivität von Studierenden in einer Fremdsprache äußert sich 
in der Teilnahme an veranstalteten kreativen Wettbewerben, themengebundenen Quizspielen, runden Tischen, an den Treffen mit Delegationen aus Ausland etc. Um das Interesse der Magisterstudenten an wissenschaftlicher Arbeit, der Abfassung der Vorträge in einer Fremdsprache, den Reden in den Studentenkonferenzen, der suche und Bearbeitung fremdsprachlicher Literatur für Magisterarbeiten $\mathrm{zu}$ steigern, werden die Studenten-Energetiker zur Mitgliedschaft in den wissenschaftlichen Zirkeln herangezogen. So funktioniert an der Nationalen Universität «Lwiwsjka Politechnika» der wissenschaftliche Zirkel «Speaking club», an der Nationalen technischen Iwan Puluj Universität Ternopil «English club», in der getrennten Abteilung der Nationalen Universität für Lebens- und Umweltwissenschaften der Ukraine «Das Bereshaner agrotechnische Institut» «Deutschsprachige Welt».

Das Erlernen einer Fremdsprache von Magisterstudenten der energetischen Studiengänge wird nicht ausschließlich durch Unterrichtsstunden und außerschulische Aktivitäten beschränkt. Der Prozess der Bildung der fremdsprachlichen Kompetenz zukünftiger Ingenieur-Energetiker geht über die Grenzen der Hochschulen hinaus. Die absolvierten Praktika durch Agrimpuls, APOLLO ermöglichen den Studierenden praktische fremdsprachliche Fertigkeiten $\mathrm{zu}$ perfektionieren und berufsbezogene Kenntnisse in einem fremdsprachlichen Raum zu erweitern. Dies wirkt sich positiv auf Steigerung der inneren Motivation von Magisterstudenten zum Fremdsprachenerwerb aus, begünstigt die Erweiterung des Gesichtskreises über ein Land, dessen Sprache erlernt wird, seine Geschichte und Kultur, Wissenschaft und Technik, gegenwärtige Lage der Energetik und Errungenschaften im Bereich alternative Energiequellen, formiert fremdsprachliche, soziokulturelle und berufsbezogene Kompetenzen der zukünftigen Ingenieur-Energetiker.

Das Niveau der internationalen Tätigkeit der technischen Hochschulen, an denen die Forschungsarbeit durchgeführt wurde, wird bei $60 \%$ von Hochschullehrern als sehr erfolgreich bewertet, $40 \%$ weisen auf die Notwendigkeit hin akademische Verbindungen mit europäischen Universitäten auszubauen. Dank der internationalen Zusammenarbeit, die auf Integration in den weltweiten Bildungsraum auf Entwicklung akademischer Mobilität, Schaffung gemeinsamer Lehr- und wissenschaftlicher Forschungslabors, Basen für Produktionspraktiken gerichtet ist, haben sich $6 \%$ zukünftiger Ingenieur-Energetiker am Studentenaustauschprogramm beteiligt, $54 \%$ von Magisterstudenten bot sich die Möglichkeit fremdsprachliche Kenntnisse, Fähigkeiten und Fertigkeiten während des Praktikums einzusetzen. Die Umfrage von $14 \%$ der Teilnehmer hat ergeben, dass sie durch das niedrige Niveau der Fremdsprachekenntnisse den Test nicht bestanden haben. Die fehlende Möglichkeit des fremdsprachlichen Aufenthaltes im Verlaufe der Berufsausbildung haben 26\% der Teilnehmer der Umfrage begründet.

Unter Berücksichtigung der internationalen Tätigkeit der technischen Hochschulen erachten wir, dass bei Berufsausbildung Studierende nicht allein eine Fremdsprache nach der Wahl (Englisch, Deutsch, Französisch) lernen sollen. Unsere Position findet Rückhalt bei $86 \%$ von Hochschullehrern, die an der Umfrage teilgenommen haben. Wir haben uns für die Meinung der Magisterstudenten zur fremdsprachlichen Vielsprachigkeit im Ausbildungsprozess interessiert. Auf die Frage: «Wie schätzen Sie solche Innovation ein, wenn an Ihrer Hochschule das Zentrum für Fremdsprachen eröffnet werden wird?» lauteten die Antworten folgendermaßen: $24 \%$ haben sich positiv geäußert, wenn nach dem vollendeten Sprachkurs die Möglichkeit bestehen würde die weltweit anerkannten Tests TOEFL, ESP, Test DaF, TEF erfolgreich $\mathrm{zu}$ bestehen und Zertifikate $\mathrm{zu}$ erhalten, die den Kenntnisstand in Englisch, Deutsch oder Französisch nachweisen; 67\% von Studierenden halten dies für eine interessante Idee, die ihre Vielsprachigkeit und Bildung der fremdsprachlichen Kompetenz begünstigen wird; nur 9\% heben hervor, dass das hauptsächlichste Augenmerk von Studierenden der energetischen Studiengänge auf das Erlernen der berufsbezogenen Fächer und nicht der Fremdsprachen gerichtet ist. Zukünftige Ingenieure des energetischen Zweiges sind sich 
der Zweckmäßigkeit und der Aktualität im Beherrschen der Fremdsprachen in gegenwärtiger Welt gut bewusst. Mit der Behauptung, dass Fremdsprachenkenntnisse die Gewähr der erfolgreichen zukünftigen Berufstätigkeit sind, sind $76 \%$ von Befragten einverstanden. Auf Grund der Beobachtung und Umfrage sieht das aufgebrachte Interesse der Studenten-Energetiker für fremdsprachliche Ausbildung folgendermaßen aus (․ㅡ. 1).

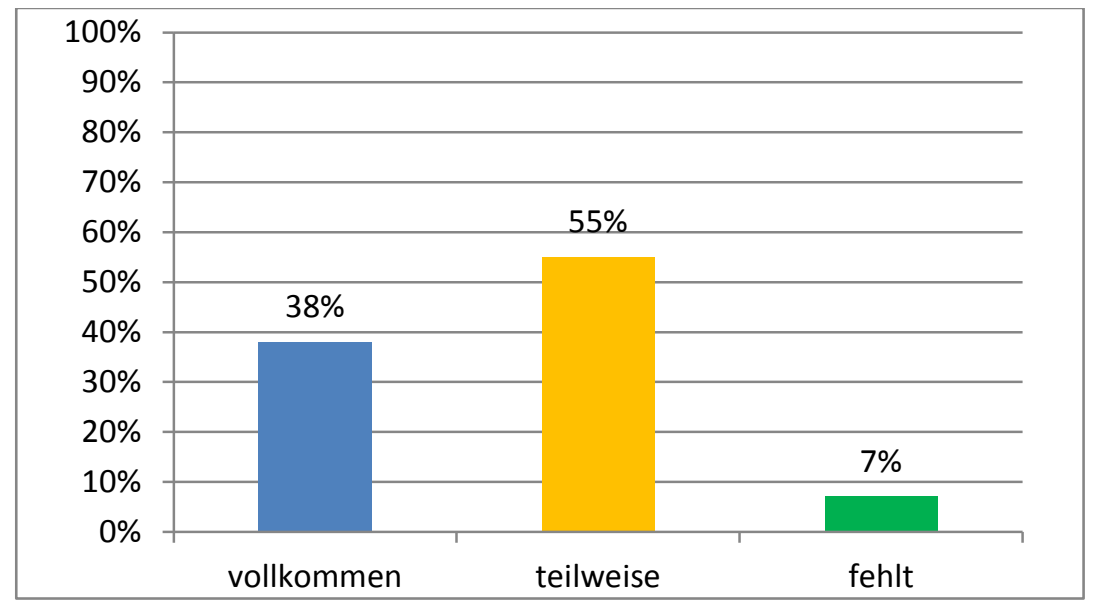

Abb. 1. Interesse der Magisterstudenten der energetischen Berufe an der fremdsprachlichen Ausbildung

Die materiell-technische Basis der Hochschulen ist einer der wichtigen Faktoren, die Einfluss auf Organisationsetappe der fremdsprachlichen Ausbildung von Studierenden ausüben. Vorhandene Sprachlabors, die mit modernen technischen Informationsmitteln ausgerüstet sind, erlauben es ein natürliches Milieu für Modellierung der kommunikativen Situationen des berufsbezogenen Charakters $\mathrm{zu}$ schaffen und erleichtern Aneignung der berufsbedingten Zielsprache von zukünftigen Ingenieur-Energetikern. Die Versorgung der Unterrichtsräume mit audio-visuellen Mitteln beurteilen 24\% der Hochschullehrer als ausgezeichnet, $14 \%$ - befriedigend. Fehlende spezialisierte Räume für fremdsprachliche Ausbildung stellen 17\% der Befragten fest. 45\% der Hochschullehrer äußerten ihre Ansicht darüber, dass Lehrzimmer für Fremdsprachen mit audio-visuellen Mitteln nur teilweise versorgt sind und der Erneuerung bedürfen.

Die nächste Etappe der Organisation der fremdsprachlichen Ausbildung hängt von der Versorgung der Studierenden mit erforderlicher Fachliteratur ab. Die Behauptungen der 17\% der Hochschullehrer zeugen davon, dass der Bücherbestand der Bibliotheken jährlich erneuert und mit aktuellen wissenschaftlich-technischen
Mitteln vervollständigt wird. Wie von $14 \%$ der Hochschullehrer gemeldet wird, tauchen in der Anschaffung der fremdsprachlichen Lehrbücher und Lehrwerke für Studierende der energetischen Fachrichtungen Schwierigkeiten auf. Im Zusammenhang damit verlegen $45 \%$ der Hochschullehrer einzeln oder in Zusammenarbeit mit ihren Kollegen gemeinsame Lehrbücher und Lehrmittel zum weiteren Gebrauch im Lernprozess. Die Lehrliteratur der ausländischen Herausgabe wird von $67 \%$ der Befragten bevorzugt. Nach unserer Auffassung kann der authentische Lernstoff ausländischer Verfasser nur jenen Studierenden angeboten werden, die Fremdsprachen in Untergruppen lernen, wobei Niveaus des Beherrschens fremdsprachlicher Kenntnisse befolgt werden.

Doch der erfolgreiche Prozess fremdsprachlicher Ausbildung hängt nicht nur von äußerlichen und innerlichen Bedingungen $\mathrm{ab}$, die sich an den technischen Hochschulen und außerhalb dieser Hochschulen bilden, sondern auch von den Teilnehmern des Lernvorgangs selbst: d.h. von den Hochschullehrern und Erwerbern der Bildungsstufe «Magister». Umfangreiches Wissen, berufliche Kompetenz, Fähigkeit für das Fach ein Interesse zu erwecken, in jedem Magisterstudenten einen 
Fremdsprachenkenner $\mathrm{zu}$ erblicken und zur aktiven Arbeit heranzuziehen, unabhängig vom Niveau des Beherrschens des fremdsprachlichen Lehrstoffes - das sind charakteristische Eigenschaften der Hochschullehrer, die sich auf die Bildung positiver Einstellung der Studierenden $\quad \mathrm{zu} \quad$ Lehrfächern «Geschäftsfremdsprache» / «Fachbezogene Fremdsprache» / «Fachorientierte Fremdsprache» auswirken, führen zur Steigerung der inneren Motivation, regen zur systematischen zielbewussten Arbeit an. Das oben erwähnte gewährleistet die Verwirklichung der rückwärtigen zwischenmenschlichen Verbindung: «Hochschullehrer-Student», «Student-Student», «Student-Hochschullehrer». Im Zusammenhang damit beruht die fremdsprachliche Ausbildung der Erwerber der Bildungsstufe «Magister» an den technischen Hochschulen auf Kompetenzansatz und personenorientiertem, wirksamem, problematischem und differenziertem Herangehen. Bei Vermittlung fremdsprachlicher Kenntnisse, Bildung von Fähigkeiten und Fertigkeiten ist das Augenmerk der Hochschullehrer auf Persönlichkeiten des zukünftigen Ingenieur-Energetikers mit seinen eigenen Motiven und Bedürfnissen, individuellen Fähigkeiten und Möglichkeiten gerichtet. Die Magisterstudenten mit verschiedenem
Wissensstand studieren doch in den Grenzen einer Untergruppe, was in der Verwirklichung des personenorientierten und differenzierten Herangehens Schwierigkeiten bereitet. Das ist einer der Hauptnachteile in der fremdsprachlichen Ausbildung der Studierenden an den technischen Hochschulen. Dank berufsmäßiger Meisterschaft und organischer Verknüpfung traditioneller Lehrmethoden mit innovativen Technologien gewährleisten Fremdsprachenhochschullehrer innere Differenzierung der Studierenden bei fremdsprachlicher Ausbildung. Der Meinung der Hochschullehrer nach sind kommunikative, situative Lernmethoden und die Arbeit in Gruppen am meisten effektiv zur Entwicklung fremdsprachlicher Fähigkeiten und Fertigkeiten der Magisterstudenten. Unten wird das prozentuelle Verhältnis der Anwendung moderner Unterrichtstechnologien von Fremdsprachenhochschullehrern im Bildungsvorgang dargelegt, die nach der Stufe der Effektivität bestimmt wurden (Abb. 2).

Die Hochschullehrer ziehen im Unterricht die Aufgaben vor, deren Lösung die Organisation der kognitiven Bildungsaktivität der Magisterstudenten laut den auf der $\underline{\text { Abb. } 3}$ dargelegten Formen voraussieht.

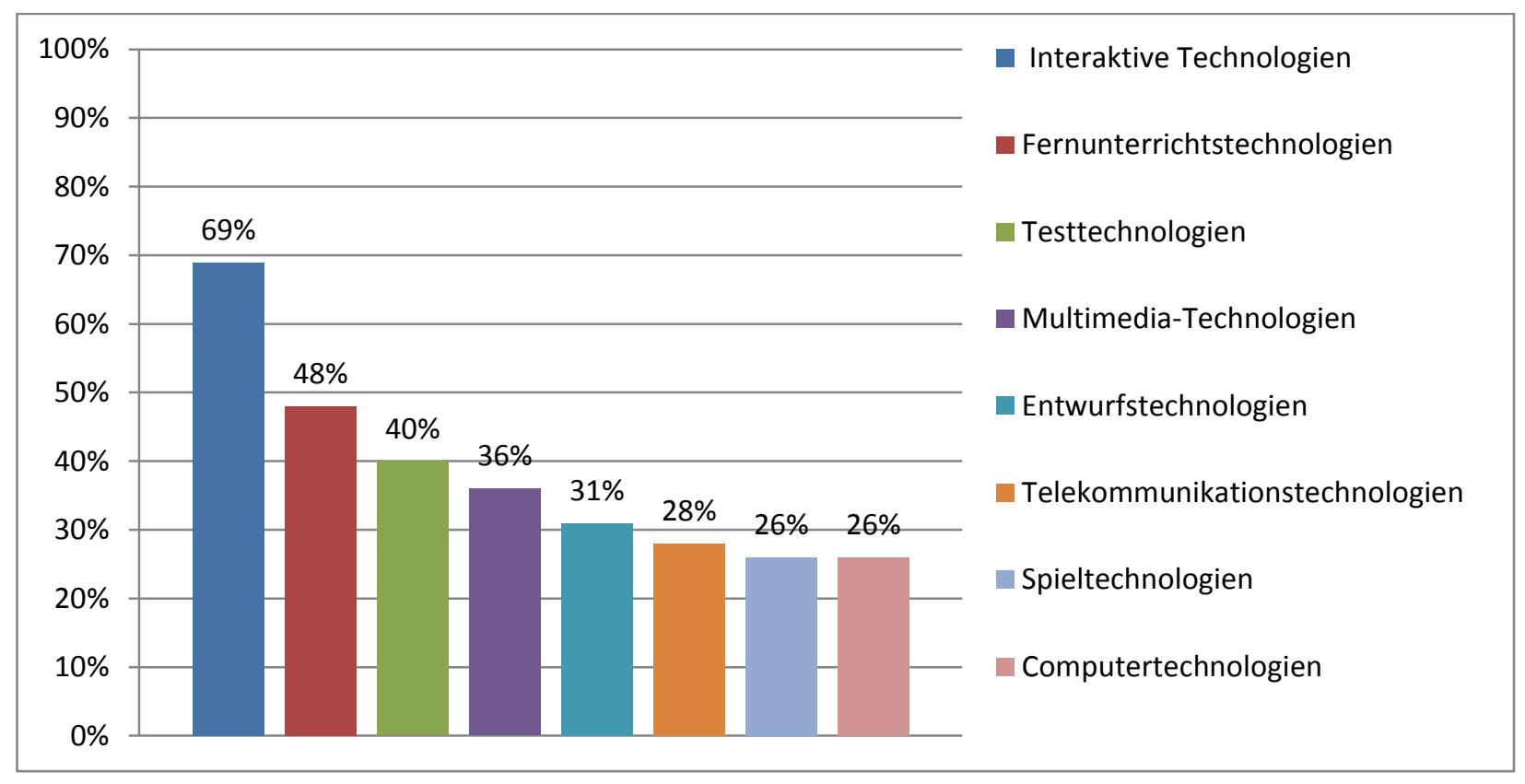

Abb. 2. Einsatz von modernen Unterrichtstechnologien in den Fächern «Geschäftsfremdsprache» / «Fachbezogene Fremdsprache» / «Fachorientierte Fremdsprache» 


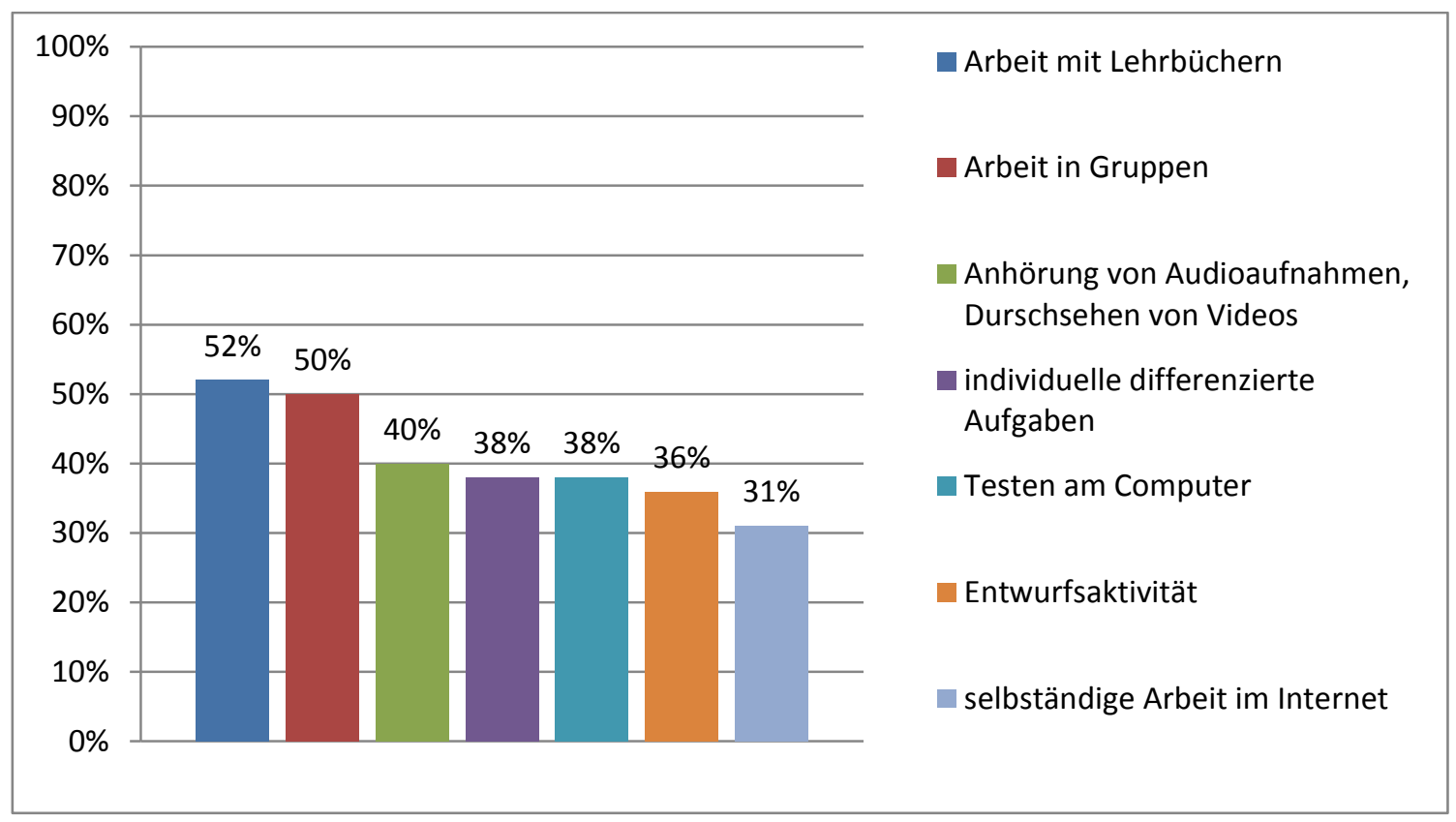

Abb. 3. Organisationsformen der Unterrichtsarbeit von Magisterstudenten im Fremdsprachenunterricht

Auf Grund der Beobachtungen haben sich Schwierigkeiten herausgestellt, die bei Magisterstudenten der energetischen Fachrichtungen während der Ausführung der oben erwähnten Aufgaben auftauchen. Laut der Stufe der Erstrangigkeit kann man sie in folgender Reihefolge vorstellen:

1) Aneignung der Grammatik der deutschen Sprache;

2) grammatische Fehler im mündlichen und schriftlichen Sprechen;

3) Einhaltung deutlicher Aussprache und des Sprechtempos;

4) sich den allgemeinen Wortschatz, technische Fachausdrücke, berufsbezogenes Vokabular einprägen und sie kommunikativ aktivieren;

5) Übersetzung von Texten aus Wissenschaft und Technik;

6) spontane Äußerung eigener Meinung unabhängig von Diskussionsthema oder Kommunikationssituationen;

7) unkonzentrierte Aufmerksamkeit, während eine fremdsprachliche Information wahrgenommen wird.

Zur Bewältigung der bestimmten Schwierigkeiten und zur Steigerung des Niveaus der fremdsprachlichen Kenntnisse bekommen 12\% der Magisterstudenten Nachhilfestunden, 11\% besuchen einen Fremdsprachenkurs, 65\% arbeiten selbständig an der Vervollkommnung von fremdsprachlichen Kenntnissen, Fähigkeiten und Fertigkeiten. 12\% von Studierenden behaupten, dass eine Fremdsprache ein zweitrangiges Lernfach ist, dessen Kenntnisse die Ingenieur-Energetiker zur Lösung der Berufsaufgaben nicht benötigen und deswegen lexisch-grammatische Fehler und deren Berichtigung nicht beachten.

Das Erlernen einer Fremdsprache ist ein komplizierter und anhaltender Prozess, der die Etappe der Entwicklung sprachlicher Fähigkeiten und Aneignung fremdsprachlicher Information auf dem Niveau des Verstehens umfasst; Möglichkeit das Lernfach selbst mit sprachlicher Einheit zu verbinden; Wahrnehmung, Begreifen und Präzisierung der Bedeutung des fremdsprachlichen Lehrstoffes, Wahrnehmung der inhaltlichen Schattierungen der sprachlichen Einheit im Verlaufe der praktischen Sprachhandlungen; Aktivierung der formierten Fähigkeiten und Fertigkeiten im fremdsprachlichen Raum (Rusalkina, 2014, S. 25). Die Entwicklung der fremdsprachlichen Fähigkeiten und Fertigkeiten erfolgt im Rahmen einer entsprechenden Thematik. In der $\underline{A b b .4}$ sind Themenarten dargelegt, an denen die 
Erwerber der Bildungsstufe «Magister» der Studiengänge Elektrotechnik 141 «Elektroenergetik, 143 «Atomenergetik», 144 «Wärmeenergetik» ein maßgebliches Interesse zeigen. Die Prioritäten werden unter Berücksichtigung persönlicher Beweggründe gesetzt, und zwar: Erfüllung beruflicher Pflichten in einem fremdsprachlichen Raum, anstreben sich mit Ausländern in Alltagssituationen $\mathrm{zu}$ verständigen, Erweiterung der soziokulturellen Kenntnisse, fehlerfreies Beherrschen einer Fremdsprache.

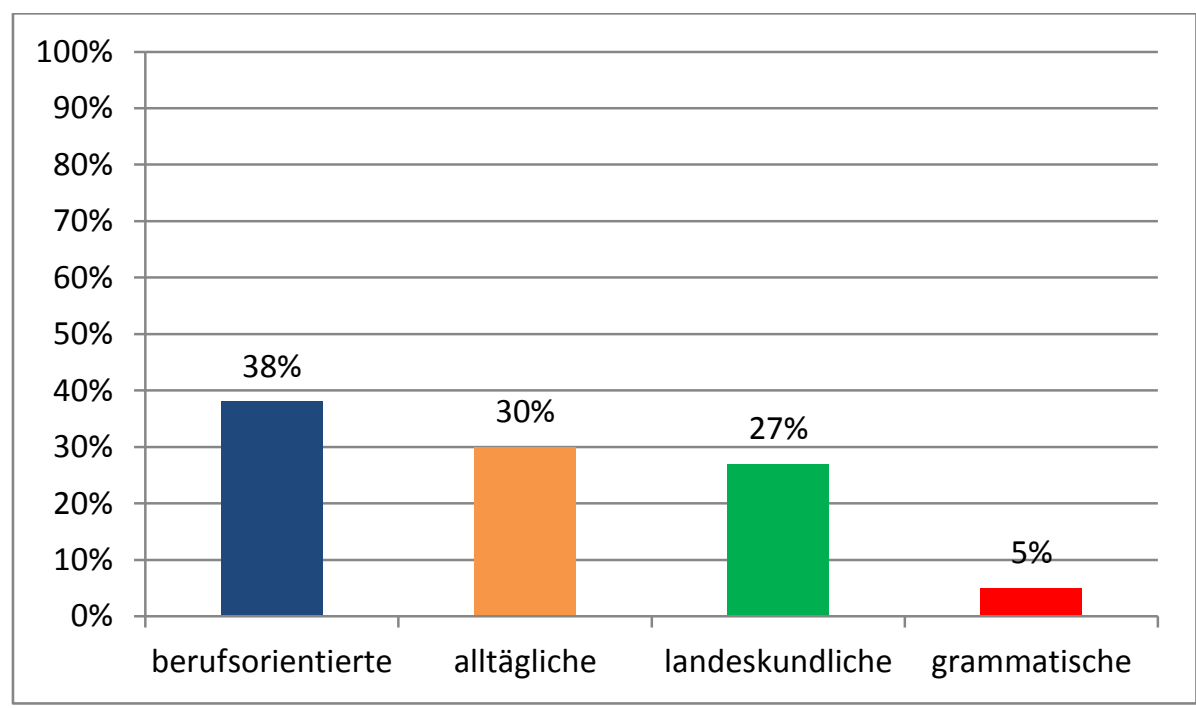

Abb. 4. Gesetzte Prioritäten fremdsprachlicher Thematik nach persönlichen Interessen und Motiven der Studenten-Energetiker

Die Themen einer fachbezogenen Fremdsprache $\mathrm{zu}$ lernen, sich anzueignen, fremdsprachlichen Lehrstoff in den praktischen Berufs- und Kommunikationssituationen einzusetzen erfolgt durch Übungs- und Festigungsaufgaben. Wir legen laut der Stufe der Erstrangigkeit die Arten von Aufgaben dar, die von Teilnehmern der Umfrage mit großem Vergnügen gemacht werden:

1) Wiedergabe der Dialoge mit verteilten Rollen;

2) Testaufgaben mit Einsatz von Computertechnologien machen;

3) Übersetzung der Texte aus Wissenschaft und Technik;

4) schöpferische Aufgaben schreiben;

5) lexisch-grammatische Übungen machen;

6) eigene Projekte schaffen und dem Auditorium darlegen;

7) fremdsprachliche Web-Seiten bearbeiten um aktuelle Information $\mathrm{zu}$ recherchieren mit weiterem Einsatz während des sprachlichen Übens;

8) Wiedergabe des Inhalts der Texte;

9) Schaffung der Präsentationen mit Einsatz von Multimedien;

10) Inszenierung der Rollen- und Geschäftsspiele;

11) Vorbereitung eines Vortrags in einer Fremdsprache.

Die erfolgreich erfüllten oben erwähnten Aufgaben tragen zur Entwicklung der Fertigkeiten im Hören, Sprechen, Lesen und Schreiben bei. Vorhandene Fähigkeit mit fremdsprachlichen Kenntnissen erfolgreich in allen Arten der Sprechtätigkeit unabhängig von Kommunikationssituationen umzugehen, drückt den Inhalt der fremdsprachlichen Kompetenz aus, die Ergebnis des Fremdspracheerlernens von Absolventen der energetischen Fachrichtungen ist. Die empirische Untersuchung hat ergeben, welcher Art der Sprechtätigkeit die Magisterstudenten-Energetiker eine besondere 
Aufmerksamkeit schenken (Abb. 5). Die gesetzten Prioritäten von Studenten sind unter Berücksichtigung der Möglichkeit der steigenden Rolle einer Fremdsprache in zukünftiger
Berufstätigkeit während der Lösung von Aufgaben des persönlichen und des ingenieurtechnischen Charakters erfolgt.

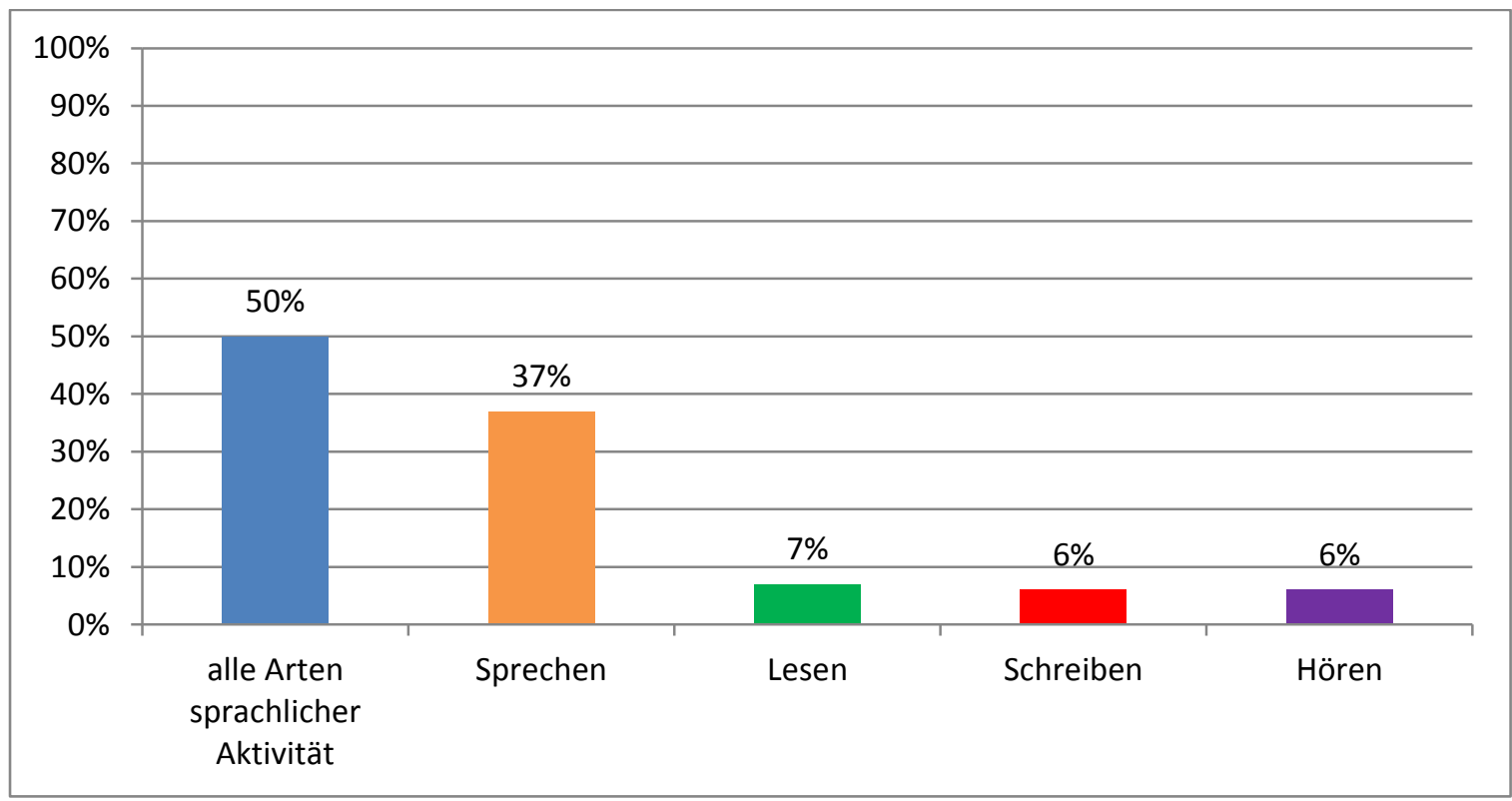

Abb. 5. Konzentration zukünftiger Ingenieur-Energetiker auf die Arten sprachlicher Aktivität

Wir meinen, dass der Entwicklung der Hör-, Sprech-, Lese- und Schreibfähigkeiten gleichmäßige Aufmerksamkeit geschenkt werden soll, ohne sie bedingt in Hauptarten und zweitrangige Arten sprachlicher Aktivität einzuteilen. Fremdsprachliche Fähigkeiten formieren sich vollständig und untrennbar unabhängig von Thematik und Zielsetzung, Organisationsformen und Ausübung fremdsprachlicher Ausbildung. Beim Spracherwerb «Geschäftsfremdsprache» / «Fachbezogene Fremdsprache» / «Fachorientierte Fremdsprache» formieren sich berufliche Fähigkeiten im Gebrauch fremdsprachlicher Kenntnisse zum Erreichen persönlicher Ziele. Am Beispiel des Themas «Dienstreise nach Deutschland» wollen wir die Möglichkeiten einer komplexen Entwicklung und Vervollkommnung von rezeptiven und produktiven Arten sprachlicher Aktivität der Magisterstudenten verfolgen, indem wir fremdsprachliche Kenntnisse des Lehrstoffes «Ein Telefongespräch», «Auf einem Betrieb», «Moderne Geschäftskorrespondenz», «Arten von Geschäftsbriefen», «Geschäftstreffen», «Eine Vereinbarung treffen» integrieren. Zum Erreichen des gesetzten Zieles werden folgende Aufgaben vorgeschlagen:

1) zwei Geschäftsbriefe schreiben: «BriefBestätigung» (allgemeine Information über eine energetische Anstalt und einen IngenieurEnergetiker, Termin und Ziel seines Aufenthaltes im Ausland), «Brief-Vorschlag» (Katalog mit Vorlagen der energetischen Ausrüstung);

2) selbständige Durcharbeitung des Textinhalts der Vereinbarung über die Einführung der entwickelten Vorlage von elektrischer Ausrüstung in Produktion;

3) Videoaufzeichnungen «Moderne energetische Anlagen» durchsehen mit weiterer Übertragung auf Kommunikation;

4) Durchführung eines Geschäftsspiels «Im Werk für Bioenergie»;

5) Schaffung und Darlegung des individuellen Projektes «Bericht über Dienstreise».

Als Ergebnis der Erfüllung der oben vorgeschlagenen Aufgaben wird der Unterrichtsstoff wiederholt und systematisiert, berufsbezogene Situationen modelliert, Fähigkeit, 
mit Hauptarten der sprachlichen Aktivität zu operieren, formiert, was die zukünftigen Ingenieur-Energetiker befähigen wird: sich im fremdsprachlichen Raum frei zu fühlen; Kontakte zu Fachleuten des energetischen Bereiches in Europa aufzunehmen, den beruflichen Wissensstand beim Lesen der wissenschaftlichtechnischen Literatur $\mathrm{zu}$ erhöhen; Videoaufzeichnungen durchzusehen; energetische Anlagen im Ausland zu besuchen; Geschäftskorrespondenz mit ausländischen Partnern auszutauschen; technische Kenndaten der elektrotechnischen und elektromechanischen Ausrüstung ukrainischer Herstellung in eine Fremdsprache zu übersetzen; Abkommen über Zusammenarbeit mit Firmen-Investoren auszuarbeiten; Ergebnisse der Projektarbeit zu beschreiben mit nachfolgender Veröffentlichung in den Fachzeitschriften im Ausland.

Im Verlauf der konstatierenden Etappe des Experiments wurden Niveaus der Bildung fremdsprachlicher Kenntnisse der zukünftigen Ingenieur-Energetiker nach allen Arten sprachlicher Aktivität ermittelt. Der qualitative und quantitative Kennwert beim Beherrschen von praktischen Fertigkeiten wie Hören, monologisches und dialogisches Sprechen, Lesen und Schreiben ist in der Abb. 6 dargelegt. Ausgehend von den durch Forschungsergebnisse erhaltenen Daten kann man sie detaillierter folgendermaßen charakterisieren.

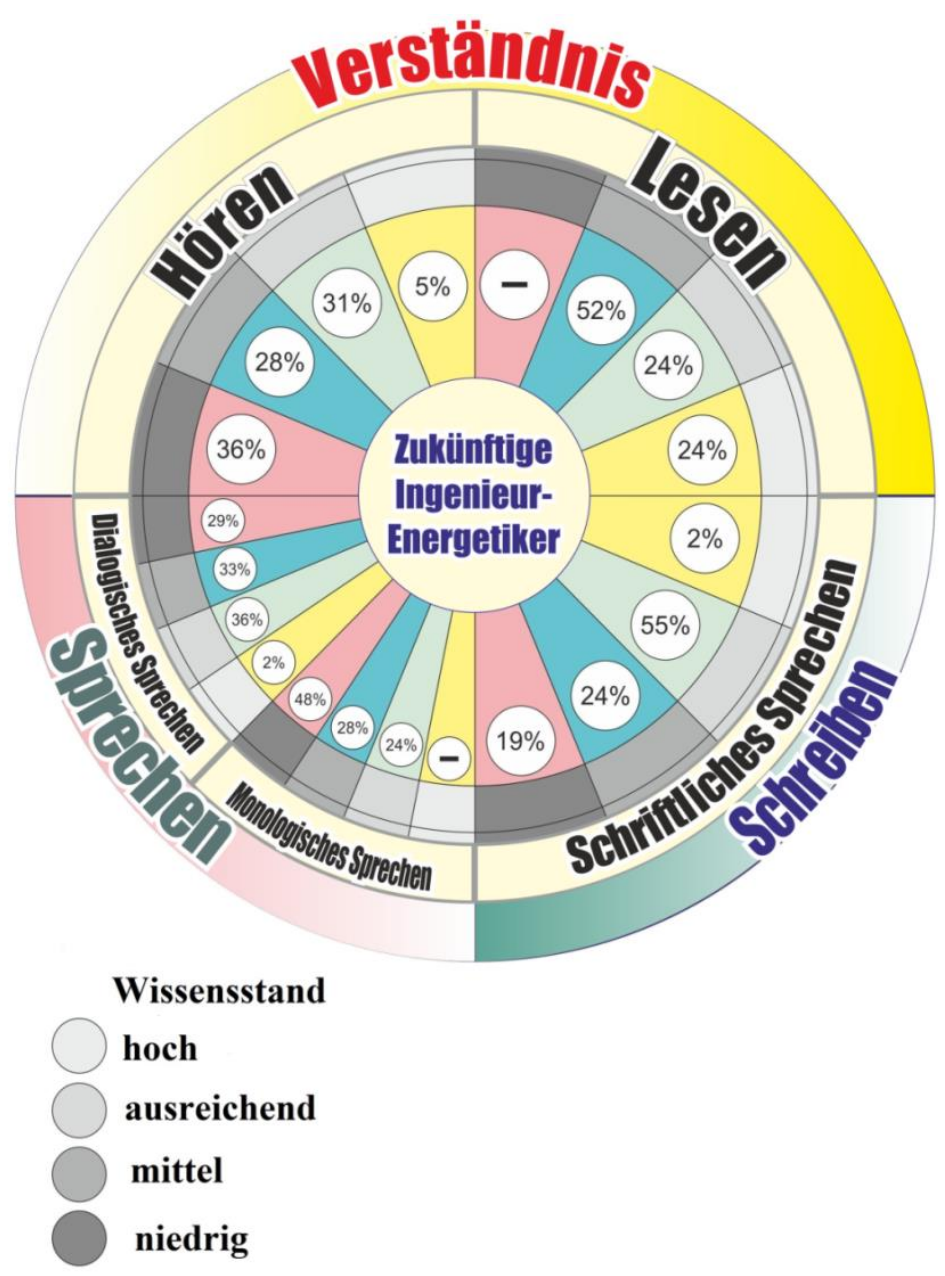

Abb. 6. Fremdsprachlicher Wissensstand zukünftiger Ingenieur-Energetiker nach Arten der sprachlichen Aktivität 
Die Grundlage für Entwicklung aller Arten der sprachlichen Aktivität bildet das Hörverständnis, ohne dessen Beherrschung es nicht möglich wäre Fähigkeiten $\mathrm{zu}$ fördern an mündlicher und schriftlicher Kommunikation aktiv teilzunehmen, Information in den fremdsprachlichen Literaturquellen $\mathrm{zu}$ finden. Fertigkeiten des fremdsprachlichen Hörens sind automatisierte Mechanismen des Hörverständnisses, die sich als Ergebnis bei mehrmaligen stereotypen Übungen im sprachlichen Vorgang formiert haben, und $\mathrm{zu}$ Fähigkeiten des fremdsprachlichen Hörens gehören Voraussicht und Prognostizierung des sprachlichen Lehrstoffes, schnelle Orientierung in seinem Inhalt, allgemeines und detailliertes Verstehen der Information, das uns bei Ermittlung von Einstellung und Absichten des Verfassers behilflich ist, und auch die Bedeutung unbekannter Wörter aus dem Kontext zu erfassen (Krasnopera, 2015, S. 16).

Wissenschaftlerin

D. Bubnowa

$(\underline{2007)}$ unterscheidet zwei Arten von Hören, die in fremdsprachlicher Geschäftskommunikation am wichtigsten sind: direktes zwischenmenschliches Zuhören in dialogischer Kommunikation und Zuhören, wenn der Zuhörer nicht über die Möglichkeit verfügt Äußerungen des Sprechenden $\mathrm{zu}$ regeln. Für die erste Art von Hören sind ein hohes Niveau des Aufschlusses von Erwiderungen des Geschäftspartners und fehlende Zeit zum Überlegen der Antwort kennzeichnend, darum ist es für deren erfolgreiche Lösung wichtig Fähigkeiten $\mathrm{zu}$ besitzen, um Sprachaktivität des Gesprächspartners zu leiten, und zwar Tonvolumen, Tempo, Wiederholung, Erläuterung. Beim Hören von Texten ohne Möglichkeit die Äußerungen des Sprechenden $\mathrm{zu}$ regeln im Zusammenhang mit fehlender Möglichkeit auf ihn einen Einfluss zu üben, und mit erhöhter Belastung des Gedächtnisses werden Fähigkeiten benötigt, bei denen es darum geht, das Gehörte im Gedächtnis zu behalten und den Textinhalt zu prognostizieren ( $\underline{\text { S. } 6})$.

Die Wahrnehmung der gehörten Information formiert im Ganzen die Vorstellung der Studierenden über Struktur und Besonderheiten einer Fremdsprache und des Landes, dessen Sprache erlernt wird. Leider nur 5\% zukünftiger Ingenieur-Energetiker verfügen über Fähigkeiten im Hören auf einem hohen Niveau, was dazu beiträgt, die gehörte Information berufsbezogenen Inhalts $\mathrm{zu}$ verstehen und wiederzugeben. 31\% der Magisterstudenten energetischer Fachrichtungen werden durch ein ausreichendes Niveau gekennzeichnet, das durch Fähigkeit bestimmt wird, den Hauptinhalt bei geschäftlichem Sprechen zu verstehen, sogar mit einer entsprechenden Anzahl von unbekannten Wörtern, die es nicht beeinträchtigen den gehörten fremdsprachlichen Stoff gedanklich zu verarbeiten. Das mittlere Niveau des formierten Hörverständnisses von kleinen nach ihrem Umfang Erzählungen, Berichten, Vorträgen, Unterhaltungen der Hochschullehrer oder Mitstudenten, Audioaufnahmen, Radio- und Fernsehsendungen ist bei $28 \%$ der Energetikstudenten zu verzeichnen. Bei 36\% der Teilnehmer des Experiments können wir bevorzugt das niedrige Niveau des Hörverständnisses feststellen, sie sind fähig nur einzelne Fragmente oder Phrasen berufsbezogener Information $\mathrm{zu}$ unterscheiden, sie sind nicht fähig sich auf die Wahrnehmung des fremdsprachlichen Sprechens $\mathrm{zu}$ konzentrieren. Wir meinen, dass die Schwierigkeiten bei Formierung des Hörverständnisses durch das schnelle Sprechtempo des Ansagers und das begrenzte technische Vokabular der Magisterstudenten verursacht sind.

Die nächste Art der sprachlichen Aktivität ist Sprechen und es ist vorrangig, es besteht aus zwei Formen: monologisches und dialogisches Sprechen. Einen konstruktiven Dialog auf einem hohen Niveau unabhängig von der Thematik zu erstellen sind fähig nur $2 \%$ der Magisterstudenten, indem sie komplizierte grammatische und lexische Konstruktionen einsetzen. 36\% der Energetikstudenten sind imstande einen Dialog zu angebotenen Themen auf ausreichendem Niveau $\mathrm{zu}$ führen. 33\% zukünftiger Ingenieur-Energetiker können sich in dialogischer Form auf mittlerem Niveau ausdrücken, indem sie einige Fehler machen, die das Verständnis und den sprachlichen Verkehr im Rahmen von gelernten Themen nicht beeinträchtigen. Die Wiedergabe eines Dialogs mit Stützung auf eine Vorlage zeugt vom niedrigen Niveau der Fähigkeiten des 
dialogischen Sprechens, was bei $29 \%$ von Energetikstudenten zu verzeichnen ist.

Einer der Mängel fremdsprachlicher Ausbildung besteht in Nichtbereitschaft zukünftiger Ingenieur-Energetiker zum monologischen Sprechen, fehlende Fähigkeit eigene Gedanken $\mathrm{zu}$ einem beliebigen berufsorientierten Thema auf einem hohen Niveau frei zum Ausdruck zu bringen. 24\% der Magisterstudenten wären bereit einen Monolog logisch, folgerichtig und informativ auf einem ausreichenden Niveau $\mathrm{zu}$ gestalten. 28\% der Energetikstudenten sind fähig fremdsprachliche Fachinformation nur mit unbedeutenden Fehlern wiederzugeben. Eine Erzählung, die wegen des geringen Wortschatzes verkompliziert wird und sich aus einfachen nichterweiterten Sätzen zusammensetzt, bei deren Bildung grammatische Fehler gemacht werden, ist ein Zeichen des niedrigen Niveaus der formierten Fähigkeiten des monologischen Sprechens, das bei $48 \%$ der Magisterstudenten energetischer Berufe vorzieht.

Lesen als eine rezeptive Art der sprachlichen Aktivität begünstigt Bereicherung und Erweiterung der Programmkenntnisse in einer Fremdsprache und im Beruf. 24\% der Energetikstudenten sind imstande authentische Fachtexte durchzuarbeiten und die nötige Information beruflichen Charakters aus fremdsprachlichen Quellen auf einem hohen Niveau auszuwählen. Das ausreichende Niveau hat sich bei $24 \%$ der Magisterstudenten formiert, das voraussieht, adaptierte Texte zu lesen und deren Inhalt vollkommen $\mathrm{zu}$ verstehen. Das Mittelniveau des Leseverständnisses zieht bei zukünftigen Ingenieur-Energetikern mit 52\% vor, die die Grundidee der nach Umfang nicht großen Texte aus Wissenschaft und Technik bestimmen und den fremdsprachlichen Lesestoff in logische inhaltliche Teile gliedern können.

Eine vernommene, gelesene, in mündlicher Form ausgedrückte fremdsprachliche Information, die zu Nachdenken, Analyse, Wiedergabe, Ausdruck eigener Meinung anregt, kann man in Schrift als lexisch-grammatische Übungen, Übersetzungen, schöpferische Aufgaben, Überlegungen, Briefe, Referate, Vorträge Annotationen, Resümees, Anzeigen etc. wiedergeben. Nur 2\% der Magisterstudenten energetischer Fachrichtungen sind befähigt sachkundig, kreativ und wissenschaftlich schriftliche Aufgaben in einer Fremdsprache mit Einsatz verschiedener Sprachmittel auf einem hohen Niveau zu machen. 55\% von Studierenden können ihre eigene Meinung in einer Fremdsprache zu einem frei angebotenen Thema, das auf die Liste der Pflichtthemen nicht kommt, auf einem ausreichenden Niveau begründen. Mit schriftlichen Aufgaben nach gelernten Themen des Inhaltsmoduls auf dem Mittelniveau kommen 24\% der Teilnehmenden am empirischen Experiment zurecht. Für 19\% der Erwerber der Bildungsstufe «Magister» ist das niedrige Niveau der Formierung von Schreibfertigkeiten kennzeichnend, das nur zur erfolgreichen Wiedergabe verschiedener Übungen nach einer Vorlage befähigt.

Aufgrund erhaltener Ergebnisse wurde eine Rangordnung der Hauptarten sprachlicher Aktivität nach Niveaus des Beherrschens der fremdsprachlichen Fertigkeiten angefertigt. Das Lesen nimmt als rezeptive Art der sprachlichen Aktivität die erste Position in der Bildung fremdsprachlicher Fertigkeiten ein, über welche die Magisterstudenten auf einem hohen Niveau verfügen. An zweiter Stelle rangieren Hören und Schreiben, welche die Studierenden auf einem ausreichenden Niveau beherrschen. Die dritte Priorität wird dem dialogischen Sprechen gesetzt und sie wird durch Mittelniveau der Bildung gekennzeichnet. Unter Hauptarten der sprachlichen Aktivität ist für monologisches Sprechen ein niedriges Niveau charakteristisch. Nachdem wir erstrangige Positionen von rezeptiven und praktischen Arten sprachlicher Aktivität nach vier Niveaus der Bildung verglichen haben, soll bedeutende Aufmerksamkeit nach unserem Erachten der Vervollkommnung der Sprechfertigkeiten einer Fremdsprache geschenkt werden. Bei Barsuk (2016) heißt es, dass fremdsprachliche Geschäftskommunikation sich im motivierten Zusammenwirken unter Repräsentanten des gemeinsamen Tätigkeitsbereiches ergibt und auf die Lösung beruflicher maßgeblicher Fragen gerichtet ist (‥19).

Die oben erwähnten ausgewerteten Qualitätsund Quantitätsmerkmale des Fremdsprachebeherrschens befähigen uns über ein befriedigendes Niveau der Bildung sowohl 
rezeptiver als auch produktiver Arten sprachlicher Aktivität zukünftiger IngenieurEnergetiker Schlüsse zu ziehen, was durch äußere und innere Aspekte fremdsprachlicher Ausbildung bedingt ist. Unserer Meinung nach sind folgende Hauptgründe zu unterscheiden:

1) fehlende wissenschaftlich begründete pädagogische Unterrichtstechnologie, die innere Motivation von Studierenden zum Erlernen der Fremdsprachen erhöhen, Behebung der Mängel im Beherrschen fremdsprachlicher Kenntnisse begünstigen, komplexe Entwicklung praktischer Fertigkeiten im Hören, Lesen, mündlichen uns schriftlichen Sprechen gewährleisten würde;
2) große Zeitspanne zwischen Erlernen der Unterrichtsfächer «Berufsorientierte Fremdsprache» (erstes Studienjahr, Bildungsstufe «Bachelor») und «Geschäftsfremdsprache» / «Fachbezogene Fremdsprache» / «Fachorientierte Fremdsprache» (fünftes Studienjahr Bildungsstufe «Magister»);

3) begrenzte Anzahl der Semester zum Erlernen einer Fremdsprache von Magisterstudenten energetischer Fachrichtungen und nicht gleichmäßige Verteilung der Unterrichtszeit zwischen Unterricht an der Hochschule und selbständiger Arbeit (Tabelle 1).

Tabelle 1. Struktur des Unterrichtspensums in einer Fremdsprache für Erwerber der Bildungsstufe «Magister» der Studiengänge 141 «Elektroenergetik, Elektrotechnik und Elektromechanik», 143 «Atomenergetik», 144 «Wärmeenergetik»

\begin{tabular}{|c|c|c|c|c|c|}
\hline \multirow[t]{2}{*}{ Technische Hochschule } & \multirow[t]{2}{*}{ Unterrichtsfach } & \multicolumn{2}{|c|}{ Stundenumfang } & \multirow{2}{*}{$\begin{array}{l}\text { Anzahl } \\
\text { im } \\
\text { Semester }\end{array}$} & \multirow{2}{*}{$\begin{array}{c}\text { Formen der } \\
\text { zusammenfassenden } \\
\text { Kontrolle }\end{array}$} \\
\hline & & $\begin{array}{l}\text { Praktischer } \\
\text { Unterricht }\end{array}$ & $\begin{array}{l}\text { Selbständige } \\
\text { Arbeit }\end{array}$ & & \\
\hline $\begin{array}{l}\text { Nationale Universität } \\
\text { «Lwiwsjka Politechnika» }\end{array}$ & $\begin{array}{l}\text { Fachbezogene } \\
\text { Fremdsprache }\end{array}$ & 30 & 60 & 1 & Vorprüfung \\
\hline $\begin{array}{l}\text { Ternopiler Nationale } \\
\text { technische Universität } \\
\text { von Iwan Puluj }\end{array}$ & $\begin{array}{l}\text { Fachorientierte } \\
\text { Fremdsprache }\end{array}$ & 56 & 64 & 1 & Vorprüfung \\
\hline $\begin{array}{l}\text { Getrennte Abteilung der } \\
\text { Nationalen Universität } \\
\text { für Lebens- und } \\
\text { Umweltwissenschaften } \\
\text { der Ukraine «Das } \\
\text { Bereshaner } \\
\text { agrotechnische Institut» }\end{array}$ & Geschäftsfremdsprache & 40 & 80 & 1 & Prüfung \\
\hline
\end{tabular}

Nachdem wir die erhaltenen Daten, aus Unterrichtsplänen und berufsbezogenen Ausbildungsprogrammen für Erwerber der zweiten Magisterstufe der Hochschulbildung im Wissenszweig 14 «Elektrisches Engineering» (Getrennte Abteilung der Nationalen Universität für Lebens- und Umweltwissenschaften der Ukraine «Das Bereshaner agrotechnische Institut», 2018a, 2018b; Lwiv Polytechnic Nationale Universität, 2016; Ternopil Ivan Puluj Nationale Technische Universität, 2016), verglichen haben, erachten wir für nötig zu bestätigen, dass 120 Stunden vollkommen ausreichend sind, um sich fremdsprachliche Fähigkeiten und Fertigkeiten anzueignen. Aber eine Fremdsprache soll zwei Semester gelernt werden, die Stundenzahl für selbständige Arbeit der Studierenden soll den Umfang praktischer Unterrichtsstunden nicht überschreiten. Es ist sinnvoll zusammenfassende Kontrolle in Form einer Prüfung durchzuführen, was das Interesse der Studierenden an der Erwerbung fremdsprachlicher Kenntnisse erhöhen und zur selbständigen Arbeit motivieren wird. Dies wird zur Behebung der Mängel bei fremdsprachlicher Ausbildung der Magisterstudenten beitragen, Bildung der Bereitschaft und Fähigkeit zukünftiger Ingenieur-Energetiker begünstigen Fremdsprachekenntnisse nach Absolvierung technischer Hochschulen auf dem Wege des 
Selbststudiums und der Selbstrealisation einzusetzen.

Aufgrund der Ergebnisse empirischer Forschung legen wir die gesamte Beurteilung des gegenwärtigen Zustandes fremdsprachlicher Ausbildung der Magisterstudenten energetischer Fachrichtungen an den technischen Hochschulen dar. Unter Berücksichtigung solcher Kriterien wie:
Organisation des Lernprozesses; materielltechnische Basis der Hochschulen; Versorgung der Unterrichtsfächer mit Lehr- und methodischen Mitteln; Studentenkontingent; internationale Tätigkeit sind die von Hochschullehrern nach einer Fünfpunkteskala gestellten Punkte auf $\underline{\mathrm{Abb} .7}$ dargelegt.

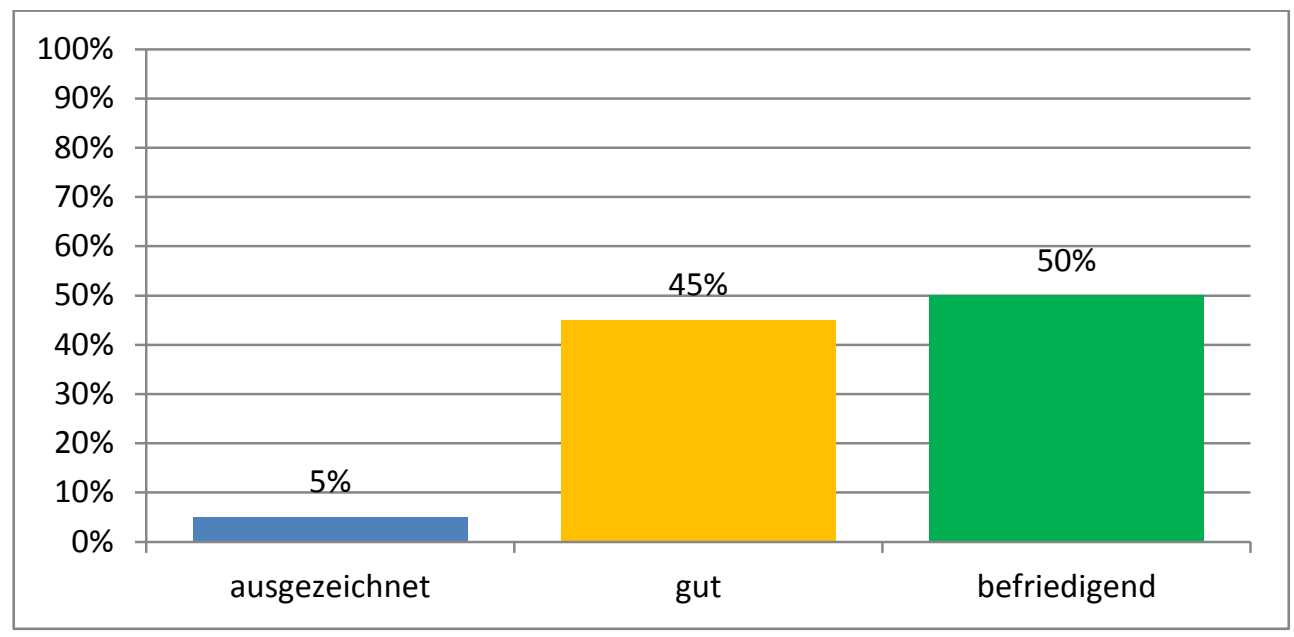

Abb. 7. Auswertung fremdsprachlicher Ausbildung der Magisterstudenten energetischer Berufe an den technischen Hochschulen

Positiv haben die Organisation fremdsprachlicher Ausbildung der Studierenden an den technischen Hochschulen Erwerber der Bildungsstufe «Magister» der Studiengänge 141 «Elektroenergetik, Elektrotechnik und Elektromechanik», 143 «Atomenergetik», 144 «Wärmeenergetik» begründet. Die Ergebnisse der Umfrage haben ergeben, dass 56\% zukünftiger Ingenieur-Energetiker mit Bildung fremdsprachlicher Kenntnisse zufrieden sind. Die Antwort «eher ja, als nein» haben 37\% der Studierenden gegeben. Doch 7\% der Teilnehmer der Umfrage meinen, dass in der Organisation des Lernprozesses Änderungen vorgenommen werden müssten. 41\% der Magisterstudenten-Energetiker behaupten, dass an den technischen Hochschulen alle erforderlichen Bedingungen für das Erlernen der Fremdsprachen geschaffen sind, 50\% sind sich dessen nicht sicher, 9\% haben eine verneinende Antwort gegeben.

Nach Auswertung des gegenwärtigen Zustandes fremdsprachlicher Ausbildung der Magisterstudenten energetischer Fachrichtungen sind wir berechtigt $\mathrm{zu}$ behaupten, dass der
Lernprozess an den technischen Hochschulen der Ukraine einer neuen Zielsetzung bedarf, das auf technisch-geisteswissenschaftliche Komponente hinzielt, auf das Verleihen einer Fremdsprache eines Status, der den Unterrichtsfächern des Zyklus beruflicher Ausbildung gleichgestellt werden kann, was die Mobilität zukünftiger Ingenieur-Energetiker bei Ausübung innovativer Tätigkeit in den heutigen Verhältnissen der reichen an Kenntnissen Gesellschaft begünstigt.

\section{SCHLUSSFOLGERUNGEN UND AUSSICHTEN FÜR WEITERE FORSCHUNGEN}

Im vorliegenden Artikel wurde die fremdsprachliche Ausbildung zukünftiger Ingenieur-Energetiker erforscht und analysiert, die eine verbindliche Komponente zielstrebigen Prozesses beruflicher Ausbildung der Studierenden energetischer Fachrichtungen an den technischen Hochschulen darstellt und auf Erreichen des Ziels im Bereich Ausbildung, Entwicklung, Erziehung, Weltanschauung und Beruf gerichtet ist. Der bestimmte Prozess wird durch Erwerbung fremdsprachlicher 
programmmäßiger bzw. allgemeiner Kenntnisse, Entwicklung praktischer Fähigkeiten und Fertigkeiten gekennzeichnet, und dient als Mittel der Bildung fremdsprachlicher Kompetenz und beruflicher bzw. persönlicher Eigenschaften der Magisterstudenten beim Erlernen des Lernfaches «Geschäftsfremdsprache» / «Fachbezogene Fremdsprache» / «Fachorientierte Fremdsprache», dessen erfolgreiche Aneignung durch Beherrschen Fremdsprachenniveaus in Übereinstimmung mit gesamteuropäischen Empfehlungen in sprachlicher Ausbildung und Bereitschaft berufliche Aufgaben in einer fremdsprachlichen Umgebung $\mathrm{zu}$ erfüllen ermittelt wird.

Nach Ergebnissen experimenteller Arbeit wurden Formen der Organisation und Durchführung fremdsprachlicher Ausbildung der Magisterstudenten an den technischen Hochschulen fundiert, die voraussehen: den kombinierten Unterricht und Sprachkurse $\mathrm{zu}$ besuchen; an den wissenschaftlichen Zirkeln, Studentenkonferenzen und kreativen Wettbewerben teilzunehmen; ein Betriebspraktikum im Ausland $\mathrm{zu}$ absolvieren. Auf der Grundlage von Beobachtung und Befragung zeigte sich positive Einstellung und bedeutendes Interesse zukünftiger IngenieurEnergetiker dem Erlernen einer Fremdsprache gegenüber, das auf kompetenzgebundenen, persönlichkeitsorientierten,

handlungsorientierten, problematischen und differenzierten Ansätzen beruht, wo der harmonische Einsatz von kommunikativen, situativen und gruppenweise Unterrichtsmethoden mitsamt innovativen pädagogischen Technologien dafür sorgen, die erwähnten Ansätze in die Wirklichkeit umzusetzen. Im vorliegenden Artikel wurden vier Stufen der formierten rezeptiven und produktiven Arten sprachlicher Aktivität der Magisterstudenten energetischer Fachrichtungen ermittelt und analysiert, die eine Grundlage fremdsprachlicher Kompetenz zukünftiger Ingenieur-Energetiker bilden und in Fähigkeit ausgedrückt werden, über Fertigkeiten beruflicher Verwendung einer Fremdsprache für die Arbeit mit wissenschaftlich-technischer Literatur und über Führung der Geschäftskommunikation in fremdsprachlicher Umgebung zu verfügen.
Die Zukunftsaussichten weiterer Forschungen sehen wir in wissenschaftlicher Begründung und methodisch ausgearbeiteter innovativer Bildungstechnologie, die fremdsprachliche Ausbildung der Magisterstudenten energetischer Fachrichtungen an den technischen Hochschulen gewährleisten und das Ergebnis bestimmten Prozesses berufliche Mobilität der IngenieurEnergetiker im zukünftigen Tätigkeitsumfeld begünstigen würde.

\section{LITERATURVERZEICHNIS}

Getrennte Abteilung der Nationalen Universität für Lebens- und Umweltwissenschaften der Ukraine «Das Bereshaner agrotechnische Institut». (2018a). Ausbildungsplan für Fachkräfte der zweiten Magisterstufe der Hochschulbildung für Studiengang 141 «Elektroenergetik, Elektrotechnik und Elektromechanik» des Wissenszweigs 14 «Elektrisches Engineering». Qualifikation: Magister für Elektroenergetik, Elektrotechnik und Elektromechanik [pdf]. Bereshany. Entnommen https://bit.ly/2UNu2rS.

Getrennte Abteilung der Nationalen Universität für Lebens- und Umweltwissenschaften der Ukraine «Das Bereshaner agrotechnische Institut». (2018b). Ausbildungs- und Berufsprogramm der Hochschulbildung «Elektroenergetik, Elektrotechnik und Elektromechanik» der zweiten Magisterstufe der Hochschulbildung für Studiengang 141 «Elektroenergetik, Elektrotechnik und Elektromechanik» des Wissenszweigs 14 «Elektrisches Engineering». Qualifikation: Magister für Elektroenergetik, Elektrotechnik und Elektromechanik [pdf]. Bereshany. Entnommen https://bit.ly/2UMttih.

Ternopil Ivan Puluj Nationale Technische Universität. (2016). Ausbildungs- und Berufsprogramm «Elektroenergetik, Elektrotechnik und Elektromechanik» der zweiten Magisterstufe der Hochschulbildung für Studiengang 141 «Elektroenergetik, Elektrotechnik und Elektromechanik» des Wissenszweigs 14 «Elektrisches Engineering». Qualifikation: Magister für Elektroenergetik, Elektrotechnik und Elektromechanik [pdf]. Ternopil. Entnommen http://m.tntu.edu.ua/ storage/pages/00000485/op141m.pdf.

Lwiv Polytechnic Nationale Universität. (2016). Ausbildungs- und Berufsprogramm zur Erlangung der Bildungsstufe «Magister» für Studiengang 141 «Elektroenergetik, Elektrotechnik und Elektromechanik» des Wissenszweigs 14 «Elektrisches Engineering». Qualifikation: Magister für Elektroenergetik, Elektrotechnik und Elektromechanik 
[pdf]. Lwiw. Entnommen http://lp.edu.ua/sites/ default/files/attach/2016/4222/tytul_programy_mag istr.pdf

Azzolini, L. S., Amelina, S. M., Hamanyuk, W. A., \& Zhdanowa, N. S. (2014). Rahmencurriculum für den Studienbegleitenden Deutschunterricht an den ukrainischen Hochschulen. Kiew: Lenwit.

Bakajewa, H. J., $\quad$ Borysenko, O. A., $\quad$ Zujenok, I. I., Iwanischtschewa, W. O., $\quad$ Klymenko, L. J., Kosymyrsjka, T. I., Kostryzjka, S. I., Skrypnyk, T. I., Todorowa, N. J., \& Chodzewa, A. O. (2005). Curriculum für kommunikatives Berufsenglisch. Kyjiw: Lenwit.

Barsuk, L. S. (2016). Pädagogische Verhältnisse der Bildung fremdsprachlichen berufsbezogenen Sprechens zukünftiger Schiffer aufgrund des kognitiven Kommunikationsansatzes. (Dissertation zur Erlangung des akademischen Grades Doktor Philosophie). Chersoner staatliche Universität, Cherson.

Bubnowa, D. W. (2007). Methodik für Geschäftskommunikation in Englischer Sprache für Studenten von oberen Studienjahren der technischen Lehranstalten. (Zusammenfassung der Dissertation zur Erlangung des akademischen Grades Doktor Philosophie). Kyjiwer Nationale linquistische Universität, Kyjiw.

Chomenko, O. W. (2012). Fremdsprachliche Ausbildung als verbindliche Komponente der Hochschulbildung unter Bedingungen der Globalisierung und Informatisierung der Gesellschaft. Hochschulbildung der Ukraine, (2), 5561.

Krasnopera, T. W. (2015). Psychologische Besonderheiten der Entwicklung des Hörverstehens zukünftiger Fachkräfte für intenationale Beziehungen. (Zusammenfassung der Dissertation zur Erlangung des akademischen Grades Doktor Philosophie). Nationalakademie für pädagogische Wissenschaften der Ukraine Institut für Psychologie von H. S. Kostjuk, Kyjiw.

Nikolajewa, S. J. (2003). Gemeinsamer europäischer Referenzrahmen für Sprachen: lernen, lehren, beurteilen. Kyjiw: Lenwit.

Nitenko, O. W. (2015). Fremdsprachliche Ausbildung der Fachkräfte der Rechtswissenschaften im dreistufigen System der Hochschulbildung: europäische Erfahrung. (Dissertation zur Erlangung des akademischen Grades Doktor der Wissenschaften). Nationalakademie für pädagogische Wissenschaften der Ukraine Institut für Hochschulbildung, Kyjiw.

Rusalkina, W. H. (2014). Fähigkeitsbildung der englischsprachigen Geschäftskommunikation bei zukünftigen Ärzten. (Dissertation zur Erlangung des akademischen Grades Doktor der Philosophie). Südukrainische nationale pädagogische Universität von Konstjantyn Uschynsjkyj, Odessa.

Schumejko, N. W. (2017). Fremdsprachliche Berufsausbildung zukünftiger Fachkräfte geisteswissenschaftlicher Spezialisierung an den Universitäten in der Slowakei. (Dissertation zur Erlangung des akademischen Grades Doktor der Philosophie). Nationalakademie für pädagogische Wissenschaften der Ukraine Institut für pädagogische Bildung und Bildung für Erwachsene, Kyjiw.

Schumsjka, O. A. (2015). Besonderheiten fremdsprachlicher Ausbildung der Fachkräfte naturwissenschaftlicher Spezialisierung an der gegenwärtigen Hochschule der Ukraine. Wissenschaftliche Zeitschrift der Universität Ushhorod. Serie: Pädagogik. Sozialarbeit, (37), 205209.

\section{REFERENCES}

Separated Subdivision of National University of Live and Environmental Sciences of Ukraine «Berezhany Agrotechnical Institute». (2018). Curriculum of specialists training Second (master) level of higher education in specialty 141 «Power engineering, electrical engineering and electromechanics» of the branch of knowledge 14 «Electrical engineering». Qualification: Master of electrical engineering, electrical engineering and electromechanics [pdf]. Berezhany. Retrieved from https://bit.ly/2UNu2rS. (in Ukrainian)

Separated Subdivision of National University of Live and Environmental Sciences of Ukraine «Berezhany Agrotechnical Institute». (2018). Educational and professional higher education program «Electric power engineering, electrical engineering and electromechanic» Second (master) level of higher education in specialty 141 «Power engineering, electrical engineering and electromechanic» of branch 14 «Electrical engineering». Qualification: Master of electrical engineering, electrical engineering and electromechanic [pdf]. Berezhany. Retrieved from https://bit.ly/2UMttih. (in Ukrainian)

Ternopil Ivan Puluj National Technical University. (2016). Educational and professional higher education program «Electric power engineering, electrical engineering and electromechanic» Second (master) level of higher education in specialty 141 «Power engineering, electrical engineering and electromechanics» of branch 14 «Electrical engineering». Qualification: Master of electrical engineering, electrical engineering and 
electromechanics [pdf]. Ternopil. Retrieved from http://m.tntu.edu.ua/storage/pages/00000485/op14 1m.pdf. (in Ukrainian)

Lviv Polytechnic National University. (2016). Educational and professional higher education program «Electric power engineering, electrical engineering and electromechanics» Second (master) level of higher education in specialty 141 «Power engineering, electrical engineering and electromechanics» of branch 14 «Electrical engineering». Qualification: Master of electrical engineering, electrical engineering and electromechanics [pdf]. Lviv. Retrieved from http://p.edu.ua/sites/default/files/attach/2016/422 2/tytul_programy_magistr.pdf. (in Ukrainian)

Azzolini, L. S., Amelina, S. M., Hamaniuk, V. A., \& Zhdanova, N. S. (2014). Frame program of German language for professional communication in Universities in Ukraine. Kyiv: Lenvit. (in Ukrainian)

Bakaieva, H. Ie., $\quad$ Borysenko, O. A., Z Zuienok, I. I., Ivanishcheva, V. O., $\quad$ Klymenko, L. Y., Kozymyrska, T. I., Kostrytska, S. I., Skrypnyk, T. I., Todorova, N. Yu., \& Khodtseva, A. O. (2005). English for Specific Purposes (ESP) National Curriculum for Universities. Kyiv: Lenvit. (in Ukrainian)

Barsuk, S. L. Pedagogical conditions of formation of foreign professional communicative skills of future navigators based on Communicative and Cognitive approaches. (Candidate of Pedagogic Sciences Thesis). Kherson State University, Kherson. (in Ukrainian)

Bubnova, D. V. (2007). Methods of teaching business interaction in English to senior students of technical institutions of higher learning. (Author's abstract of Candidate of Pedagogic Sciences Thesis). Kyiv National Linguistic University, Kyiv. (in Ukrainian)
Khomenko, O. V. (2012). Foreign language education as an integral component of higher education in the conditions of globalization and informatization of society. Vyshcha osvita Ukrainy, (2), 55-61. (in Ukrainian)

Krasnopera, T. V. (2015). Psychological Peculiarities of Listening Skills Development of International Relations Future Specialists. (Author's abstract of Candidate of Psychology Sciences Thesis). G. S. Kostyuk Institute of Psychology of the National Academy of Pedagogical Sciences of Ukraine, Kyiv. (in Ukrainian)

Nikolaieva, S.Yu. (2003). Common European framework of reference for languages: learning, teaching, assessment. Kyiv: Lenvit. (in Ukrainian)

Nitenko, O. V. (2015). Foreign language training of the future lawyers in frame of the three-cycle higher education system: European experience. (Doctor of Pedagogic Sciences Thesis). Institute of Higher Education of the National Academy of Pedagogical Sciences of Ukraine, Kyiv. (in Ukrainian)

Rusalkina, L. G. (2014). Developing Business English Communicative Skills in Future Doctors. (Candidate of Pedagogic Sciences Thesis). K. Ushynsky South Ukrainian National Pedagogical University, Odessa. (in Ukrainian).

Shumeiko, N. V. (2017). Professional Foreign Language Training of Future Specialists in Humanities at the Universities of Slovakia. (Candidate of Pedagogic Sciences Thesis). Institute of Pedagogical and Adult Education of the National Academy of Pedagogical Sciences of Ukraine, Kyiv. (in Ukrainian)

Shumska, O. A. (2015). Features of foreign language training of specialists of natural history cycle at modern higher institutions of Ukraine. Naukovyi visnyk Uzhhorodskoho universytetu. Seriia: Pedahohika. Sotsialna robota, (37), 205-209. (in Ukrainian)

\section{Über den Autor:}

Natalia Bilan, Hochschullehrerin des Lehrstuhles für Geisteswissenschaften, Getrennte Abteilung der Nationalen Universität für Lebens- und Umweltwissenschaften der Ukraine «Das Bereshaner agrotechnische Institut» (Akademisch st., 20, Bereshany, Ternopil Region, Ukraine, 47501), ORCID: http://orcid.org/0000-00029996-8593, Researcher ID: E-9782-2019, natalja_bilan@ukr.net

About the author:

Nataliia Bilan, Senior Lecturer of the Humanitarian Disciplines Department, Separated Subdivision of National University of Live and Environmental Sciences of Ukraine "Berezhany Agrotechnical Institute" (Akademichna str., 20, Berezhany, Ternopil Region, Ukraine, 47501), ORCID: http://orcid.org/0000-0002-99968593, Researcher ID: E-9782-2019, natalja_bilan@ukr.net 